\title{
HACIA MODELOS DE GESTIÓN INNOVADORES EN LA ADMINISTRACIÓN PÚBLICA: EL CASO DE LA EMPRESA PÚBLICA DE CORREOS CHILE
}

\author{
Susana Villanueva \\ susana.villanueva@mail.com \\ Consultora internacional
}

La innovación ha adquirido gran relevancia durante el último tiempo, sirviendo de marco para implementar modelos de gestión de calidad adecuados a la naturaleza de las organizaciones públicas, contribuyendo así a la generación de valor. En este artículo, se aborda el caso de la empresa pública Correos Chile, analizando su modelo de gestión a la luz de los principales modelos de gestión de calidad existentes. Cinco variables constituyen el foco de análisis utilizado, a partir de los cuales se identifican elementos propios de la gestión de calidad de esta Empresa.

Palabras claves: Innovación, gestión pública, empresas públicas, Correos Chile.

\section{TOWARDS INNOVATIVE MODELS OF MANAGEMENT IN PUBLIC ADMINISTRATION: THE CASE OF CHILE'S Public POSTAL SERVICE}

Innovation has become increasingly relevant in recent years as a framework for the implementation of quality management models suited to the nature of public organizations, generating value. This article examines the case of Chile's Public Postal Service, analyzing its management model in light of mainstream quality management models. The analysis focuses on five variables, through which the main elements of the Postal Service's quality management system are identified.

Keywords: Innovation, public management, quality management, public enterprises, Postal Service of Chile. 


\section{INTRODUCCION}

En las últimas décadas, el concepto de innovación ha adquirido gran relevancia en la gestión pública. Es así como la tendencia de incorporar Modelos de Gestión de Calidad en las organizaciones públicas se ha considerado como un esfuerzo por encontrar fuentes de innovación que, inspiradas desde la empresa privada, pueda ser adaptada para permitir la creación de valor para los diferentes grupos sociales involucrados como trabajadores, clientes, proveedores y la sociedad en general. Como señala Elizabeth Oria (2008), las empresas públicas han incorporado conceptos e instrumentos de la empresa privada tales como empleado, empresa, cliente, calidad, innovación, mercado, productividad, transformación, cambio, lo que les ha servido de marco para la incorporación de mejoras organizativas a nivel de procesos, estructuras y, muy especialmente, estrategias.

En este contexto, el presente artículo pretende contribuir al desarrollo del conocimiento sobre las estrategias de innovación de las empresas públicas a partir del enfoque del nuevo Modelo de Negocio de la Empresa Pública de Correos Chile, empresa con una larga historia de servicio, enmarcada en un amplio proceso de transformaciones que han ido desde un correo tradicional con escasas perspectivas de crecimiento y supervivencia, hacia un cambio en su forma de gestión y que durante los últimos años se ha visto enfrentada a una creciente competencia en todas sus áreas y a un mercado postal que cambia rápidamente por la incursión de nuevas tecnologías de información.

Tomando en cuenta lo anterior, surgen elementos que hacen atractivo llevar adelante un estudio de caso para una empresa pública de estas características. Entre ellas interesa saber: ¿Cómo impacta en la gestión de la empresa pública de Correos Chile el asumir principios de gestión de calidad y cómo repercute en la aplicación de este modelo? ¿Qué características, criterios o principios de calidad hay en el modelo de negocio de la empresa pública de Correos Chile que le permitan asemejarse o aproximarse a los criterios de modelos de gestión de calidad como Gerencial Deming, Malcom Baldrige, EFQM e Iberoamericano para la Excelencia?

Este artículo propone un modelo que guía el análisis en torno a los elementos que deben ser considerados para implementar un modelo de gestión de calidad 
en empresas públicas. Este modelo se nutre tanto de elementos teóricos como empíricos, aportando así a un mayor conocimiento y desarrollo conceptual sobre la innovación que surge de la gestión de calidad. Para ello se utilizará como fuente de información fundamental las entrevistas en profundidad, que fueron aplicadas a directivos de la Empresa Correos Chile.

Este artículo es un estudio de caso, donde la unidad de análisis es la Empresa Pública de Correos Chile. La utilización del estudio de caso tiene el objetivo de profundizar en las implicaciones que tiene para una organización la decisión de adoptar principios y criterios de un modelo de Gestión de Calidad. Según Rialp (1998), el estudio de caso está siendo cada vez más aceptado como instrumento de investigación científica en el área del management, sobre todo al comprobarse que el acceso a información de primera mano y la comprensión de los procesos de toma de decisión, implementación y cambio en las organizaciones requiere de un tipo de análisis no realizable con la suficiente profundidad a través del estudio de un número elevado de observaciones.

De acuerdo a la definición de Yin (1994, pág. 13), un estudio de caso es una investigación empírica que estudia un fenómeno contemporáneo dentro de su contexto de la vida real. Hay muchas maneras de explicar el mundo de las organizaciones, el método del caso en la investigación, resulta atractivo por ser exhaustivo y riguroso, adopta en general una perspectiva integradora y permite indagar detalladamente y con mayor profundidad que los estudios estadísticos. Su ámbito de aplicación está bien definido, estudiando temas o fenómenos contemporáneos sobre los cuales el investigador no tiene control.

Como la investigación es de tipo analítico-explicativo, se han escogido métodos cualitativos intentando analizar y explicar los criterios que han llevado a la empresa pública de Correos Chile adoptar un modelo de negocio en la gestión de la empresa que le permita mantenerse y competir en un mercado cada vez más competitivo. Asimismo, se analizan las líneas de acción y características organizacionales que facilitan o restringen a la empresa pública de Correos Chile adoptar criterios y principios de un modelo de gestión de calidad. Por otro lado, se encuentra el análisis de corte transversal, mediante el cual se lleva a cabo la comparación entre el modelo de negocio de la empresa pública de Correos Chile con los criterios de excelencia de un modelo de calidad/excelencia en gestión. 
La recopilación de información se realiza a través de la revisión de fuentes primarias y secundarias. Asimismo se hicieron entrevistas a informantes clave de los diferentes niveles jerárquicos de la organización; esto nos permitirá obtener información cualitativa más puntual sobre temas y aspectos que se están investigando. Kvale (1996) señala que el propósito de la entrevista de investigación cualitativa es obtener descripciones del mundo vivido por las personas entrevistadas, con el fin de lograr interpretaciones fidedignas del significado que tienen los fenómenos descritos.

De otro lado Galindo (1998), manifiesta que la entrevista es un excelente instrumento heurístico para combinar enfoque prácticos, analíticos e interpretativos implícitos en todo proceso de investigación. Es a través de ellas que las percepciones, las actitudes y las opiniones de los informantes clave, que no pueden inferirse de la observación, se hacen entonces accesibles. Para ello se realizaron entrevistas a ejecutivos, gerentes, jefes, supervisores, administrativos, operadores y carteros de Correos Chile. Por medio de esta técnica se obtuvo el relato de las experiencias del proceso de orientación hacia nuevas formas de trabajo, nuevos enfoques de gestión, cambios en la cultura organizacional, implementación del nuevo modelo de negocio de la empresa, entre otros. El tamaño de la muestra se ha establecido en base al criterio de "saturación", esto es, el momento en que la información comienza a ser redundante, no aportando ningún nuevo punto de vista analítico, por lo que se decide la conclusión de la recogida de información.

Las secciones siguientes presentan una breve discusión conceptual sobre innovación y gestión de calidad, con un foco preponderante en las empresas públicas, donde se identificarán las variables relevantes para proponer un modelo de análisis. La aplicación del modelo permitirá evidenciar similitudes y diferencias entre nuestro caso de estudio y otros modelos existentes y experiencias. 
I. LA MODERNIZACiÓN DE LA GESTIÓN PÚbliCA COMO MARCO DE LA INNOVACIÓN EN LA GESTIÓN DE CALIDAD EN LAS EMPRESAS PÚBLICAS

\section{LA GERENCIA PÚBLICA COMO MARCO DE LA GESTIÓN DE CALIDAD}

De acuerdo con Castelló Taliani y Lazaro Álvarez (1998), en el ámbito de la Administración Pública la búsqueda de la calidad no puede considerarse como algo nuevo, lo que ha variado es la orientación que se le ha dado a lo largo del tiempo, siendo posible distinguir tres grandes etapas: una primera fase, en la que prima el respeto a las normas y los procedimientos; una segunda en la que se asimila la calidad y la eficiencia, y la tercera fase en la que se entiende a la calidad como la satisfacción de las necesidades de los ciudadanos a través de la innovación (Beltrami, 1992).

Ante estos nuevos enfoques, el paradigma burocrático orientado hacia el apego de reglas y procedimientos, su alejamiento de las necesidades de los clientes y sus escasas responsabilidades con relación a los resultados y mecanismos de decisión, acciones basadas en el secreto, la obediencia, la especialización y el monopolio, aparece como altamente inadecuado para enfrentar las necesidades de una gestión eficiente en tiempos de cambio.

La burocracia pasa, entonces, a ser vista no sólo como un impedimento a la gestión eficiente, sino también, como un actor entregado a sus intereses corporativos en detrimento de los intereses colectivos. Es así como existe un consenso sobre la necesidad de reformar la administración pública, siendo el principal referente la "Nueva Gestión Pública", que propone "una serie de cambios intencionales de las estructuras y procesos de organizaciones del sector publico con el objetivo de que funcionen mejor en algún sentido" (Pollit y Brouckaert, 2004).

Por otra parte, esta necesidad de cambio es identificada por Crozier (1997) como resultado de la incapacidad para responder frente a los cambios y orientarse hacia una nueva forma de gestión y dominar la complejidad creciente de las actividades colectivas. Para enfrentarse a la complejidad actual, es necesario que el gerente tenga libertad para tomar decisiones rápidas, lo que requiere más conocimiento e información, más discreción, autonomía, flexibilidad, responsabilización y compromiso con relación a los resultados. La nueva gerencia pública constituye 
un modelo de gestión que combina los papeles de empresario y gerente público. Entraña un modelo empresarial de manejo del sector público, pues la expresión inglesa public management es el reflejo de la inserción de la perspectiva gerencial dentro del sector público (Savoie, 1995), significando la implantación de la imagen empresarial dentro de organizaciones o empresas públicas.

Este modelo de gestión centra su énfasis en la incorporación de herramientas de gestión empresarial en la que su justificación central es la necesidad de recrear en el ámbito público condiciones similares a las del funcionamiento de los mercados. En efecto la mayor parte de las tecnologías sugeridas aparecen como respuestas creadas y desarrolladas por el sector privado frente a las exigencias del mercado y obedecen a preocupaciones tales como la tasa de rentabilidad, obtención de resultados, costos, inversiones, el grado de competitividad, la orientación al cliente y la preocupación por la eficiencia, en tanto criterios que empresarios y gerentes deben constantemente promover y desarrollar para asegurar la adaptación de sus organizaciones a los entornos cada vez más competitivos (López, 2002).

En consecuencia se requiere de un cambio orientado al gerenciamiento de lo público, estos cambios representan un nuevo orden, significan la transformación de la cultura del sector público, la revalorización del papel del gobierno, nuevas formas de asegurar la provisión de los servicios públicos y forjar nuevas relaciones en la participación del poder a diferentes niveles, donde la devolución de autoridad gerencial es una piedra angular de las formas de mejorar el desempeño en el ámbito público (Guerrero, 2004).

Como actores relevantes del desarrollo de la administración pública, las empresas públicas han debido enfrentar importantes desafíos en este sentido. A la hora de establecer estrategias de mejora en la empresa pública, el profesor de economía del IESE, Xavier Vives, propone seguir los principios que guían a las privadas, pero adaptados a las especificidades de la empresa pública y al sector público en general. Vives reconoce que, a diferencia del sector privado, el gobierno de la empresa pública se enfrenta a tres retos: definir de manera precisa la función de propietario dentro de la administración; mejorar su transparencia, tanto de los objetivos como de los resultados de la empresa; y dar capacidad de decisión a los gestores y consejos de administración, proponiendo cinco ideas para superar las dificultades y mejorar la gestión de la empresa pública: Establecer objetivos claros; 
introducir incentivos basados en la disciplina de mercado; aislar de la influencia política al consejo de administración y los gestores; ofrecer información suficiente (transparencia) tanto por parte de la empresa como del Gobierno y establecer un grado de competencia suficiente en el mercado del producto.

\section{GESTIÓN DE CALIDAD: HACIA UNA CLARIFICACIÓN CONCEPTUAL}

Es sabido que la mejora sólo es concebible desde el conocimiento previo de lo que se está haciendo y se está logrando, la mejora de la gestión pública es en gran medida, mejora de las organizaciones públicas. Por lo tanto, para mejorar la gestión pública es necesario no sólo evaluar el resultado de políticas o programas, sino conocer, analizar y evaluar el funcionamiento de las organizaciones y empresas públicas.

La gestión de calidad ha dejado de ser dominio exclusivo de las empresas privadas, modelos que logran altos resultados en sus operaciones comerciales ya que la necesidad de ofrecer servicios que cumplan con estándares de calidad y a satisfacción del usuario se viene trasladando al ámbito de lo público. El concepto de calidad en la gestión ha experimentado transformaciones desde un enfoque restringido y orientado al control final de los productos y al aseguramiento de los procesos de producción hasta un planteamiento integral que involucra a todos los niveles y áreas en una organización; en otras palabras, si anteriormente la gestión de calidad tenía como parámetros el producto elaborado y el proceso de fabricación, en los últimos ańos el parámetro de la gestión de calidad total o excelencia es la propia organización en su conjunto (Garde, 2005).

El ámbito en el que opera la gestión de calidad ya no es un ámbito delimitado por las relaciones internas en un departamento o entre el productor o consumidor, es un ámbito multidimensional que contempla todas las interrelaciones de la organización y de ésta con todos los grupos de interés. En definitiva, la calidad ha evolucionado de una concepción inicialmente tecnocrática y comercial a una concepción democrática y social (Garde, 2005). La calidad, como concepto de gestión y sus herramientas asociadas constituyen activos de primera importancia para perseguir objetivos de mayor eficiencia en la prestación de servicios, pero la calidad no puede definirse exclusivamente como satisfacción del cliente, sino como satisfacción de todos los grupos de interés (stakeholders); evidentemente, 
alcanzar logros excelentes con respecto a un público objetivo más complejo comporta el alineamiento de toda la organización como un sistema integrado.

Los conceptos de calidad aunque prestados del mundo de la empresa privada, han ido en evolución, así en la Carta Iberoamericana del 2008 se define el concepto de la calidad en la gestión pública como una cultura transformadora que impulsa a la Administración Pública a su mejora permanente para satisfacer progresivamente las necesidades y expectativas de los ciudadanos, al servicio público, con justicia, equidad, objetividad y eficiencia en el uso de los recursos públicos. El concepto de calidad siempre está en constante evolución, desde la aplicación a conceptos industriales hasta su aplicación a empresas de servicio en general (Gurú, 2003; Albacete, 2004; Albacete et al., 2007). En el contexto de las organizaciones industriales, desde el comienzos del siglo pasado, se entendía la calidad como: "El grado en que un producto cumplía con las especificaciones técnicas que se le habían establecido cuando fue diseñado" (Crosby, 1979). Más recientemente el concepto de calidad transcendió hacia todos los ámbitos de la organización definiéndola como: "Todas las formas a través de las cuales la organización satisface las necesidades y expectativas de sus clientes, sus empleados, las entidades implicadas y toda la sociedad en general" (Albretch, 1994).

Las teorías de calidad se han desarrollado en los países más avanzados como Estados Unidos y Japón. Los principales autores y precursores estadounidenses de los conceptos de la calidad total son: Philip B. Crosby, Edward W. Deming, Armand V. Feigenbaum y Joseph M. Juran. Dentro de los autores japoneses las ideas de: Kaoru Ishikawa, Shigeru Mizuno, Shigeo Shingo y Geinichi Taguchi. Estos son sólo los más importantes que han publicado literatura en el tema de la calidad total pues, sin lugar a dudas, son los que aportan la mayoría de las ideas originales en esta área del conocimiento.

\section{GESTIÓN DE CALIDAD EN EMPRESAS PÚBLICAS: DESAFÍOS PARA SU IMPLEMENTACIÓN}

Realizar una gestión de calidad en las empresas públicas se está convirtiendo en una prioridad en muchos países donde el sector público todavía ocupa un peso importante de la economía. Según la Organización para el Desarrollo de la Cooperación Económica (OCDE), el buen gobierno de las empresas públicas es fundamental para garantizar la eficiencia económica y la competitividad globales de 
un país. Pero, ¿qué estrategias hay que seguir para conseguirlo? Para responder a esta pregunta, el profesor Xavier Vives analiza los principales problemas de la empresa pública y propone posibles estrategias de mejora, basadas en las directrices reconocidas internacionalmente elaboradas por la OCDE.

Según el profesor Vives, los problemas de la empresa pública son mayores que los de la empresa privada por varias razones. En primer lugar, mientras la empresa privada tiene un solo objetivo central de maximización de valor, la pública tiene objetivos múltiples .

Por otro lado, las empresas públicas llevan asociado el problema de doble agencia. Esto es, a los conflictos habituales en las corporaciones privadas, derivados del comportamiento interesado de los gestores en relación al propietario, la empresa pública debe lidiar con el comportamiento interesado de los políticos o burócratas en relación a la ciudadanía. Ello puede derivar tanto en un excesivo intervencionismo político como en pasividad excesiva por parte del Estado. Cuando una empresa pública compite en el mercado de productos o servicios con otras empresas, debe mantener niveles de eficiencia aceptables, de lo contrario incurriría en pérdidas sistemáticas que pueden hacerla política y técnicamente inviable.

Un tercer problema de las empresas públicas deriva de los conflictos de interés que se suscitan por el papel del Estado como propietario de la empresa pública a la vez que regulador y comprador o suministrador de bienes y servicios de la empresa, ello puede llevar a la existencia de discriminación entre empresas o tratos de favor. Por otra parte, en el año 2005 la OCDE publicó un conjunto de directrices sobre gobierno corporativo de la empresa pública, para garantizar eficiencia y una mejor transparencia en dicho sector. Este informe enfatiza la importancia de establecer un marco legal y regulador claro para la actuación de las empresas públicas, adaptándose en la mayor medida posible a las directrices corporativas de las empresas privadas para evitar distorsiones en el mercado y favorecer la competencia en igualdad de condiciones con la empresa privada ${ }^{2}$.

1 Los cuales de ser compatibilizados, cuando distintas partes interesadas entran en conflicto, pueden llegar a ser contradictorios y generar dificultades para la evaluación de resultados.

2 Implica una separación clara entre los diferentes papeles que el Estado ejerce en los mercados en que participa como propietario: como propietario de la empresa y como regulador del mercado. En relación a la financiación, se recomienda evitar situaciones en que el Estado asuma la responsabilidad de la deuda. 
El Estado, como accionista mayoritario debe actuar de manera activa y clara en sus funciones de propietario. Según la OCDE, hay que evitar tanto que el Estado adopte una posición pasiva en el control de las empresas públicas como que se involucre en la gestión diaria de las mismas. El organismo afirma que la mejor forma de control es mediante una entidad centralizada que defina, de manera clara y transparente, los objetivos del Estado como propietario, así como la política general y su implementación. Esta entidad es la que debería responder ante el Parlamento. La OCDE también afirma que la empresa pública debe reconocer explícitamente sus compromisos en relación a todas las partes interesadas (stakeholders) así como la posible influencia de estos en los procesos de decisión de la empresa.

Para que esta transparencia sea real, la OCDE recomienda hacer públicos los informes sobre distintos aspectos de la empresa, en particular los que puedan suponer un conflicto de interés potencial y la realización de auditorías tanto internas como externas, y es la agencia que coordina la propiedad pública de empresas quien debe informar con informes de manera permanente. Por último, y para mejorar la calidad de los consejos de administración de las empresas públicas, la OCDE recomienda que los consejos deben contar con el poder necesario para controlar la gestión de la empresa de manera independiente a la política del Gobierno. Para esto se debe desarrollar una estructura y un sistema de nominación de los miembros donde predomine la objetividad, la profesionalidad e independencia, buscando atraer a los profesionales más cualificados.

\section{Principales MOdelos De GeStión De CALIDAD: BASES PARA UN ANÁLISIS}

Como respuesta a tiempos de intenso cambio y competencia, las organizaciones se han visto obligadas a aumentar su eficacia y eficiencia, desarrollar sus diferentes capacidades organizacionales y agregar valor a sus productos o servicios. Para esto, han tenido que revisar y reestructurar sus procesos clave, desplegar su imaginación y anticiparse a las futuras demandas de la sociedad (Mergan et al., 2000). Los Modelos de Calidad Total en la Gestión son referentes que ayudan a las organizaciones, midiendo en qué punto se encuentran dentro del camino hacia la excelencia, sirven como marco de trabajo referencial y como una herramienta de 
diagnóstico organizacional, habiéndose convertido en valiosos instrumentos de desarrollo e incentivo para el alcance de la competitividad organizacional.

Un estudio realizado en el año 2000 por el Dr. Vinod Singhal del Instituto Tecnológico de Georgia y el Dr. Kevin Hendricks de la Universidad Western Ontario, de más de 600 ganadores de premios a la calidad, mostró que, en conjunto, experimentaron incrementos o mejoras significativas en el valor de sus acciones ordinarias, ingresos de explotación, ventas, retorno sobre ventas, empleo y ganancia neta.

Existen diversos modelos pero en todos ellos subyacen los principios básicos de la Gestión de Calidad Total, es decir, las mejores prácticas en el ámbito de la gestión de las organizaciones. Bajo este contexto, analizaremos los modelos de gestión de calidad, partiendo de los conceptos sensibilizadores de calidad de los modelos como: Modelo Gerencial Deming, Modelo Malcom Baldrige, Modelo European Foundation for Quality Management (EFQM), Modelo Iberoamericano para la Excelencia; ya que la administración pública moderna se ve obligada a adoptar distintas herramientas de gestión utilizadas por las empresas privadas; obedeciendo a la necesidad de no solo ser eficaces en la gestión sino también eficientes, y esta eficiencia alude directamente a la relación costo beneficio de los emprendimientos, porque no solo importa lograr los resultados sino también interesa con qué costo se logra. Por tanto las organizaciones públicas tienen el desafío de prestar servicios innovadores con eficacia, eficiencia y con calidad.

En términos generales, si se analizan las principales variables que componen estos modelos, se pueden encontrar los siguientes elementos. En primer lugar, todos los modelos consideran al liderazgo como un criterio fundamental y de primer orden.

En primer lugar, el modelo Deming está orientado a generar un engranaje organizativo basado en la cooperación interna y externa donde el aprendizaje se logra a través de un trabajo en conjunto con un enfoque a implementar prácticas de gestión de procesos y mejora continua. Este modelo de calidad se desarrolla en Japón en 1951 por la JUSE (Unión Japonesa de Científicos e Ingenieros) y recoge la aplicación práctica de las teorías japonesas del Control Total de la Calidad (TQC) o Control de la Calidad en toda la empresa.

La mejora continua de procesos, productos y servicios, así como la satisfacción del trabajador, son fundamentales para la satisfacción del cliente y para la su- 
pervivencia de la organización. Los valores y conceptos centrales del modelo se encuentran comprendidos en los siguientes siete criterios: a) Liderazgo visionario; b) Cooperación interna y externa; c) Aprendizaje; d) Gestión de procesos; e) Mejora continua; f) Satisfacción del empleado; g) Satisfacción del cliente. En la teoría del modelo de Deming, se establecen catorce principios que están referidos a los puntos que deben ser adoptados por los directivos y sobre los cuales se debe ejercer actuación para transformar las empresas y enrumbarlas hacia la calidad, con la finalidad de lograr los siguientes propósitos: permanecer en el negocio, proteger la inversión así como los puestos de trabajo.

En segundo lugar, el Modelo Malcon Baldrige que se sustenta en los recursos internos se fundamenta en el saber hacer y la gestión del conocimiento con el fin de guiar la mejora y la competitividad organizativa, ejerciendo un liderazgo orientado al cliente, y en el benchmarking como herramienta para mantener ventajas competitivas en la organización. Este modelo surge en la década de los 80 , como respuesta a los avances e invasión de productos japoneses en el mercado estadounidense que amenazaban fuertemente con copar el mercado americano e internacional, desplazando a las tradicionales empresas locales. El modelo Malcom Baldrige es una herramienta para la evaluación, mejora y planificación hacia la gestión de excelencia y su utilidad para las empresas se debe fundamentalmente a su orientación a resultados, el fuerte énfasis en la necesidad de desarrollar alineamiento organizacional, el decidido enfoque en los clientes y la preeminencia del concepto de focalización en todos los procesos, áreas y actividades claves.

En tercer lugar, el modelo EFQM es un modelo de autoevaluación que permite analizar las personas, los procesos y los resultados de una organización. Se basa en una serie de criterios, a los cuales hay que llegar a través de sistemas de organización definidos y correctamente implantados, con la finalidad de poder demostrar el cumplimiento de dichos criterios. Por este motivo se debe desarrollar un Sistema de Gestión de la Calidad que nos permita alcanzar los objetivos propuestos (www.raitec.es).

El modelo EFQM tiene como objetivo ayudar a las organizaciones (empresariales o de otros tipos) a conocerse mejor a sí mismas y en consecuencia, a mejorar su funcionamiento. Es un modelo que trata de medir la excelencia de las organizaciones a través de nueve criterios. Se basa en la premisa de que los resultados 
excelentes con respecto al rendimiento, clientes, personal y sociedad se logran a través del liderazgo, el personal, la política y estrategia, las alianzas y los recursos y los procesos. Muchas empresas están tomando este modelo europeo como herramienta básica para la evaluación y mejora de su gestión y resultados. Este modelo ofrece un marco genérico de criterios que pueden aplicarse ampliamente a cualquier organización. Los criterios se agrupan en dos clases: agentes facilitadores y resultados. Los criterios del 1 al 5 (agentes facilitadores) reflejan cómo la organización actúa. Los criterios del 6 al 9 (resultados) tratan de lo que ha alcanzado la organización y de lo que está alcanzando gracias a la gestión de los agentes facilitadores.

En cuarto lugar, el Modelo Iberoamericano de Excelencia en la Gestión fue implantado por la Fundación Iberoamericana para la gestión de la calidad (FUNDIBEQ) en 1999; es un modelo supranacional que trata de crear un punto de referencia único en el que se encuentren reflejados los distintos modelos de excelencia nacionales de los países iberoamericanos. Este modelo es aplicable a empresas privadas e instituciones públicas de todo tipo de sector de actividad y tamańo, y sirve como referente para evaluar una organización, establecer planes de progreso, identificar sus puntos fuertes y áreas de mejora, y así mismo es una poderosa fuente de información para el desarrollo y la planificación estratégica. Sirve como referencia para el Premio Iberoamericano de la Calidad, por ello, la Fundación Iberoamericana para la Gestión de la Calidad (FUNDIBEQ), propietaria del Modelo, lo desarrolla, actualiza y difunde. FUNDIBEQ participa en el GEM (Global Excellence Model Council) como responsable del Modelo Iberoamericano de Excelencia en la Gestión.

Existen diversas similitudes entre estos modelos. Por ejemplo, el modelo Iberoamericano es muy parecido en su esencia al modelo EFQM, ambos modelos sustentan que para lograr mejores o peores resultados existen agentes facilitadores que permiten la consecución de estos. Por otra parte, el criterio de resultados en la sociedad no es considerado en el modelo Deming pero sí en los modelos Malcon Baldrige, EFQM e Iberoamericano para la Excelencia; esto hace notar que sus enfoques tienen una orientación más ética y de responsabilidad social, como consecuencia un mayor respeto por el entorno. 
En definitiva, estos modelos nos servirán de base para construir un modelo de análisis del caso de la Empresa Correos Chile, para evidenciar similitudes con alguno de ellos o verificar que se trata de un modelo de gestión de calidad con características propias.

\section{Antecedentes para un estudio de caso: la empresa pública de Correos Chile}

\section{CARACTERÍSTICAS DE LA EMPRESA}

La empresa pública de Correos Chile es una organización con una larga historia de servicio, enmarcada en un amplio proceso de transformaciones que han ido desde un correo tradicional con escasas perspectivas de crecimiento hasta un cambio en su forma de gestión.

El 8 de febrero de 1982, mediante DFL $N^{\circ} 10$, del 24 de diciembre de 1981, del Ministerio de Transportes y Telecomunicaciones, Subsecretaría de Telecomunicaciones, se pone fin al antiguo Servicios de Correos y Telégrafos creándose la "Empresa de Correos Chile", como un organismo de administración autónoma del Estado, con patrimonio propio, y se relaciona con el gobierno a través del Ministerio de Transportes y Telecomunicaciones, quien ejerce la función reguladora (DFL N $\left.{ }^{\circ} 10\right)$.

Desde el año 2001, por instrucciones del Ministro de Transportes y Telecomunicaciones, el Directorio de Correos Chile reporta sobre su gestión al Sistema de Empresas Públicas (SEP). Mediante DFL Nº 22, del Ministerio de Hacienda, de mayo de 2003, se determinó que los directores de Correos serán designados por el Consejo del SEP, organismo que ejerce así en plenitud el rol de representante del dueño, el Estado de Chile, mientras el Ministerio de Transportes y Telecomunicaciones ejerce su rol de regulador, por medio de la Subsecretaría de Telecomunicaciones (Memoria SEP 2008). Correos Chile es una persona jurídica de derecho público, creada por Decreto con Fuerza de Ley $N^{\circ} 10$ de 24 de diciembre de 1981, publicado en el Diario Oficial del 30 de enero de 1982; texto legal que constituye su ley orgánica. 
Correos Chile ejecuta sus funciones como empresa comercial, con un régimen jurídico de derecho público y se administra en forma autónoma. Para ello cuenta con patrimonio y personalidad jurídica propios y se relaciona con el gobierno central a través del Ministerio de Transportes y Telecomunicaciones, Subsecretaría de Telecomunicaciones. Por disposición de su ley orgánica, está sujeta a la fiscalización de la Contraloría General de la República. La empresa está dirigida y administrada por un Directorio de cinco miembros, designados por S.E. el Presidente de la República, de los cuales uno actúa como Presidente y otro como Vicepresidente.

\section{UNA GESTIÓN QUE APUESTA POR EL CAMBIO}

Correos Chile es una empresa con una larga historia de servicio, que durante los últimos años se ha visto enfrentada a una creciente competencia en todas sus áreas. De un pasado monopólico, pasaba a enfrentarse a nuevos competidores con gran variedad de empresas y servicios en el rubro y en un mercado cada vez más competitivo. Es en este contexto que la empresa tuvo que enfrentarse a un mercado postal con cambios vertiginosos por la aparición de nuevas tecnologías de información. Tras la crisis del año 2000, la empresa de Correos Chile tuvo que enfrentar grandes cambios y desafíos mayores en materia de comunicaciones. Era necesario mejorar la dañada imagen al interior de la organización y reconstruir la confianza de los trabajadores hacia sus directivos como condición mínima para comenzar a trabajar.

Se iniciaron los grandes cambios siendo necesario construir una visión para Correos que resultase atractiva para sus clientes, para sus dueños y para sus trabajadores: una organización ejemplo de empresa pública confiable, rentable, moderna y con una dirección profesional y visionaria, en la que fuese un orgullo trabajar, teniendo como objetivo que esta visión se posicione poco a poco en las mentes y corazones de los trabajadores de la empresa. Durante el periodo 2001 - 2002 se colocaron los pilares para la estructuración de un nuevo enfoque de negocio, centrándose en la generación de condiciones para incrementar los ingresos por ventas en un ambiente cada día más competitivo, y en reducir drásticamente sus costos operativos. 
Este periodo estuvo marcado por el rediseño de todos los procesos de negocio de la empresa, se dio inicio a la generación de un flujo constante de proyectos que permitiesen la consolidación de los rediseños y que se vieron potenciados por los avances logrados en los sistemas de información de la empresa. El rediseño de los procesos (procesos postales, incluyendo el marketing y la venta, admisión en agencias, clasificación, transporte y logística, distribución y retorno, facturación y cobranzas, parrilla de productos, diversificación de negocios, soporte administrativo y de sistemas, control y relaciones laborales) partió con la especificación de la cadena de valor de Correos Chile, un concepto clave que describe las principales actividades en las que la compañía debe generar valor para sus clientes.

\section{El nacimiento de la Estrategia Correos Chile}

A mediados de 2001, Correos Chile estableció algunos consensos básicos acerca de la situación de la empresa y su funcionamiento, permitiendo orientar el nuevo enfoque de gestión. La información recolectada durante ese año determinó que la coyuntura era favorable al cambio, que el núcleo de la crisis estaba en las personas y en la estructura, tecnología, estrategia u otras consideraciones (Informe de Gestión 2001-2003 Correos Chile).

La profesionalización de las gerencias regionales fue el punto de partida de un proceso que abordó la baja calificación del personal, jefaturas sin definiciones claras ni metas medibles.

Por último, la estructura organizacional observaba una mala coordinación entre unidades comerciales y operativas, provocando como resultado que la empresa no cumplía las promesas que ofrecían sus servicios y que muchos proyectos se duplicaban por descoordinación y desconocimiento. Durante 2001 el Directorio privilegió la formación de un equipo ejecutivo seleccionado a través de empresas externas, mediante sistemas objetivos de designación y con rentas vinculadas a resultados.

Se incorporó una innovación clave de planificación estratégica y control de gestión: el Balanced Scorecard (BSC), herramienta que enlazaba la estrategia con cuatro variables clave de desempeño gerencial: resultados financieros, satisfacción 
de los clientes, eficacia operativa y aprendizaje y crecimiento. Esta herramienta ha presentado una importante ventaja en su capacidad de identificar los factores que crean valor económico a largo plazo, facilitando la evaluación de la estrategia aplicada y orientando sus ajustes.

En este contexto general, y buscando mejorar la posición de Correos Chile en el mercado postal y superar la situación en la que se encontraba la empresa, se estableció a principios de 2001 un Plan Estratégico, cuyos fines fueron recuperar la imagen de calidad en el mercado, mejorar la rentabilidad y lograr mayor bienestar para los trabajadores de la compañía. Con la finalidad de lograr que la empresa mejorara su posición en el mercado postal, este Plan Estratégico se sustentó en siete ejes estratégicos:

a) Reafirmar la dimensión ética como valor fundamental de la empresa,

b) Mejorar el clima laboral,

c) Orientar la empresa hacia el cliente,

d) Mejorar la gestión,

e) Optimizar el uso del marco normativo postal,

f) Lograr un Código Postal de uso universal y generalizado en el país,

g) Establecer un convenio de desempeño de largo plazo con el gobierno.

Cuatro de estos ejes estratégicos se enfocaron a diseñar el camino para la superación de la crisis, otros dos estuvieron orientados a la necesidad de tomar el control por parte de la nueva administración y uno de ellos se relacionó con las condiciones del mercado. Es así que Correos Chile enfrentaba un desafiante horizonte hacia el futuro planteándose cuatro grandes objetivos estratégicos: en la División Postal, consolidar y expandir la participación de mercado en el segmento de empresas, con especial atención en los grandes clientes y en la pequeña y mediana empresa (pyme); en la División Courier, Expresos y Paquetería (CEP), aumentar la participación de mercado hasta alcanzar una posición de liderazgo, lo cual requiere de capacidad y flexibilidad para articular ofertas a la medida de cada cliente, implementar un modelo de excelencia operacional, y optimizar la gestión de personas. 


\section{El Nuevo Modelo de Negocio de Correos Chile}

Es para Chile y en particular para Correos Chile, un desafío adoptar estándares mundiales de administración de empresas, incorporando las "buenas prácticas" en esta materia sugeridas por la Organización para la Cooperación y Desarrollo Económico (OCDE) y adoptar principios de calidad en su gestión que se han constituido en las bases para la consecución de una empresa competitiva y con visión de futuro. Adoptar estos principios de gestión de calidad en el modelo de negocio ha llevado a que Correos Chile en 2008 venda cerca de $\$ 80.000$ millones y mantener más de 5 mil puestos de trabajo a lo largo del país; además de haber generado un número importante de empleos indirectos a través de los subcontratos con empresas de transporte, tecnología y otros (Memoria de Correos Chile 2008).

Fruto de un profundo trabajo colectivo de planificación estratégica realizado durante los últimos años, el año 2008 se inició el proceso de implementar en toda la organización la nueva estrategia de negocios 2008-2012 de “intimidad con el cliente”, que plantea como desafío el estrechar la relación con los clientes para entender y responder mejor que nadie a sus necesidades; para ello la empresa estará orientada a consolidar su estructura comercial y operativa, definir planes de inversiones para mejorar la competitividad, aseguramiento de procesos logísticos y de gestión a lo largo de todo el país y el aseguramiento de los ingresos de la empresa.

Para conocer el Modelo de Negocio de la empresa de Correos Chile, es necesario analizar la evolución de los criterios y principios en los que la organización ha basado y centrado su proceso de gestión de calidad. Dentro del proceso de grandes cambios y transformaciones realizados por la empresa, se puede visualizar e identificar una orientación de trabajo hacia una gestión respaldada y definida en siete aspectos: liderazgo, clientes, gestión de recursos humanos, planificación estratégica, gestión de procesos, información y conocimiento, responsabilidad social empresarial y resultados.

Todos estos elementos deberán ser analizados a la luz de los nuevos modelos de gestión de calidad, en torno a criterios similares que faciliten la identificación de parámetros comunes y que sustenten la consideración de la Empresa Pública Correos Chile como una experiencia innovadora. 


\section{Desarrollo del caso}

Tomando como base lo desarrollado en la conceptualización de las páginas precedentes así como los aportes que surgen desde la literatura, se ha construido un modelo de análisis compuesto por cinco variables. Dichas variables son:

1. Misión

2. Enfoque

3. Sistema de retroalimentación

4. Criterios

5. Conceptos principios fundamentales.

Para ello se realiza un análisis comparativo de los cuatro Modelos Internacionales de Gestión de Calidad Total: Gerencial Deming, Malcom Bardrige EFQM, Modelo Iberoamericano para la Excelencia, y el nuevo Modelo de Negocios implementado por la empresa de Correos Chile, con la finalidad de comparar y resaltar qué variables, criterios y conceptos han incidido en la instauración de un sistema de calidad en la empresa durante los últimos años, así como conocer qué similitudes o diferencias existen con los modelos de calidad internacional. Con ello se pretende identificar el modelo de gestión de calidad para una empresa pública, el que puede ser utilizado como marco para el desarrollo de investigaciones posteriores en otras empresas públicas.

A continuación se analizan de manera individual cada una de las cinco variables mencionadas:

\section{AnÁlisis Comparativo de Misión}

El Modelo Gerencial Deming, tiene como finalidad generar un sistema organizativo que fomente la cooperación tanto a nivel interno como externo en la organización, permitiendo a través de esto mejores prácticas en la gestión de procesos, mejora continua, satisfacción del cliente y por ende la satisfacción de los clientes. El Modelo EFQM se fundamenta en que la consecución de excelentes resultados con respecto a clientes, personal y sociedad, se logran a través del liderazgo, el personal, la política y estrategia, alianzas y recursos y los procesos. 
El Modelo Malcom Baldrige también se basa en una política de liderazgo para alcanzar los mejores resultados, a similitud con el Modelo Iberoamericano de la Excelencia que tiene una posición bastante similar en torno a cómo se logran excelentes resultados en una organización, con la diferencia de que además de un liderazgo debe darse una estrategia o estilo en la dirección.

De acuerdo a la información obtenida y analizada de la empresa de Correos Chile, su Modelo de Negocio tiene una orientación clara sobre la importancia del liderazgo, aspecto en el cual se puede observar que la empresa tiene una política de liderazgo orientada a dos vertientes, Liderazgo de Dirección Superior y Liderazgo de la Excelencia, siendo su principal metodología para mejorar el sistema de liderazgo, por parte de la dirección superior, la utilización de la información entregada por los estudió de clima organizacional realizados anualmente. De igual manera orientan su política de liderazgo hacia la búsqueda de un trabajo de excelencia organizacional, aspecto donde orientan sus acciones en diferentes aspectos que se destacan en el capítulo anterior.

\section{Tabla 1. Análisis Comparativo de Misión}

\begin{tabular}{l|l|l|l|l}
\multicolumn{1}{c|}{$\begin{array}{c}\text { Modelo } \\
\text { Deming }\end{array}$} & \multicolumn{1}{|c|}{$\begin{array}{c}\text { Modelo } \\
\text { Malcom } \\
\text { Baldrige }\end{array}$} & \multicolumn{1}{c|}{$\begin{array}{c}\text { Modelo } \\
\text { EFQM }\end{array}$} & \multicolumn{1}{c}{$\begin{array}{c}\text { Modelo } \\
\text { Iberoamericano }\end{array}$} & \multicolumn{1}{c}{$\begin{array}{c}\text { Modelo } \\
\text { Correos Chile }\end{array}$} \\
$\begin{array}{l}\text { Crear un sistema } \\
\text { organizativo } \\
\text { basado en la } \\
\text { cooperación } \\
\text { interna - externa } \\
\text { y mejora conti- } \\
\text { nua. }\end{array}$ & $\begin{array}{l}\text { La misión se } \\
\text { basa en un siste- } \\
\text { ma de liderazgo } \\
\text { y planificación } \\
\text { estratégica. }\end{array}$ & $\begin{array}{l}\text { Alcanzar la ex- } \\
\text { celencia a través } \\
\text { del liderazgo, la } \\
\text { política, estra- } \\
\text { tegia, alianzas, } \\
\text { recursos y pro- } \\
\text { cesos. }\end{array}$ & $\begin{array}{l}\text { Alcanzar la exce- } \\
\text { lencia a través del } \\
\text { liderazgo con un } \\
\text { estilo de dirección } \\
\text { y procesos ade- } \\
\text { cuados. }\end{array}$ & $\begin{array}{l}\text { Política de } \\
\text { liderazgo orien- } \\
\text { tada hacia la } \\
\text { excelencia en la } \\
\text { Dirección Su- } \\
\text { perior y recurso } \\
\text { humano. }\end{array}$ \\
\hline $\begin{array}{l}\text { Dirigido a resul- } \\
\text { tados en clientes } \\
\text { y empleados. }\end{array}$ & $\begin{array}{l}\text { Dirigido a re- } \\
\text { sultados para } \\
\text { clientes, recurso } \\
\text { humano, empre- } \\
\text { sarial y responsa- } \\
\text { bilidad social. }\end{array}$ & $\begin{array}{l}\text { Dirigido a } \\
\text { resultados para } \\
\text { clientes, recur- } \\
\text { so humano, } \\
\text { empresarial y } \\
\text { sociedad. }\end{array}$ & $\begin{array}{l}\text { Dirigido a resulta- } \\
\text { dos para clientes, } \\
\text { recurso humano, } \\
\text { empresarial y } \\
\text { sociedad. }\end{array}$ & $\begin{array}{l}\text { Dirigido a } \\
\text { resultados para } \\
\text { clientes, recur- } \\
\text { so humano, } \\
\text { empresarial y } \\
\text { responsabilidad } \\
\text { social. }\end{array}$ \\
\hline
\end{tabular}

Fuente: Elaboración propia basada en Nieto C. y Ros McDonnell. 


\section{ANÁlisis Comparativo: ENFOQUe}

El modelo Gerencial Deming basa su enfoque en el control estadístico, en la resolución de problemas y en la mejora continua. A diferencia del Modelo Malcon Baldrige que se fundamenta en el liderazgo hacia el cliente, en el apoyo a la organización, en la medición de índices o parámetros y en el benchamarking como herramientas para mantener ventajas competitiva en la organización.

Los modelos EFQM e Iberoamericano basan sus enfoques en determinados agentes facilitadores de la organización y estos llevan en mayor o menor grado a la consecución de resultados.

El Modelo de Negocio de Correos Chile está fuertemente definido y orientado a un liderazgo enfocado hacia la búsqueda de la satisfacción de sus clientes, a la optimización de los procesos (excelencia operacional), capacitación y productividad del recurso humano y como consecuencia a mejores éxitos comerciales.

La orientación al cliente es un pilar esencial en el enfoque del nuevo Modelo de Negocio de Correos, dirigido a orientar toda la cadena de valor de la empresa al cliente, lo que se está llevando a cabo a través de una oferta de servicios integrales.

La excelencia operacional es otro de los pilares del modelo de negocio de la empresa, que implica concentrarse en el negocio clave (servicios de logística liviana) y externalizar con expertos los otros servicios, promoviendo una actitud de excelencia en cada equipo de trabajo, fundada no sólo en el reconocimiento y la renta variable sino también en la motivación intrínseca asociada al trabajo bien hecho. Asimismo hay un enfoque hacia una gestión profesional y comprometida con las personas, esto implica el dar la más alta prioridad al desarrollo del recurso humano, principal activo de la empresa. Capacitación intensiva y masiva orientada a elevar las competencias de todo el personal, en especial de operadores, cajeros y carteros. 
Tabla 2. Análisis Comparativo Enfoque

\begin{tabular}{|c|c|c|c|c|}
\hline $\begin{array}{l}\text { Modelo } \\
\text { Deming }\end{array}$ & $\begin{array}{l}\text { Modelo } \\
\text { Malcom } \\
\text { Baldrige }\end{array}$ & Modelo EFQM & $\begin{array}{c}\text { Modelo } \\
\text { Iberoamericano }\end{array}$ & $\begin{array}{l}\text { Modelo Correos } \\
\text { Chile }\end{array}$ \\
\hline $\begin{array}{l}\text { Control } \\
\text { estadístico }\end{array}$ & $\begin{array}{l}\text { Liderazgo hacia } \\
\text { el cliente }\end{array}$ & $\begin{array}{l}\text { Los agentes } \\
\text { facilitadores: } \\
\text { liderazgo, polí- } \\
\text { tica y estrategia, } \\
\text { personas, alian- } \\
\text { zas, recursos y } \\
\text { procesos. }\end{array}$ & $\begin{array}{l}\text { Los procesos } \\
\text { facilitadores: } \\
\text { Liderazgo, estilo } \\
\text { de dirección, } \\
\text { política y estrate- } \\
\text { gia, desarrollo de } \\
\text { personas, recur- } \\
\text { sos, asociados y } \\
\text { clientes. }\end{array}$ & $\begin{array}{l}\text { Políticas adecuadas } \\
\text { para la satisfacción de } \\
\text { sus clientes. } \\
\text { La orientación al } \\
\text { cliente es un pilar } \\
\text { fundamental en el } \\
\text { enfoque del nuevo } \\
\text { modelo de negocio. }\end{array}$ \\
\hline $\begin{array}{l}\text { Resolución } \\
\text { de problemas }\end{array}$ & $\begin{array}{l}\text { Medición } \\
\text { de índices o } \\
\text { parámetros }\end{array}$ & $\begin{array}{l}\text { Conducen a } \\
\text { resultados en: } \\
\text { Clientes, perso- } \\
\text { nas, sociedad y } \\
\text { resultados clave. }\end{array}$ & $\begin{array}{l}\text { Conducen a } \\
\text { resultados en: } \\
\text { Clientes, perso- } \\
\text { nas, sociedad y } \\
\text { resultados glo- } \\
\text { bales. } \\
\end{array}$ & $\begin{array}{l}\text { Orientación al cliente } \\
\text { como pilar funda- } \\
\text { mental en el enfoque } \\
\text { del nuevo modelo de } \\
\text { negocio }\end{array}$ \\
\hline $\begin{array}{l}\text { Mejora } \\
\text { continua }\end{array}$ & Benchamarking & & & $\begin{array}{l}\text { Con la optimización } \\
\text { de los procesos, ca- } \\
\text { pacitación y produc- } \\
\text { tividad del recurso } \\
\text { humano se logran } \\
\text { mejores éxitos empre- } \\
\text { sariales y económicos. }\end{array}$ \\
\hline
\end{tabular}

Fuente: Elaboración propia basada en Nieto C. y Ros McDonnell.

\section{AnÁlisis Comparativo: Sistema de Retroalimentación}

Según Nieto C. y Ros McDonnell (2006), el sistema de retroalimentación que se establece para los modelos de gestión de calidad Deming, Baldrige, EFQM e Iberoamericano son muy similares. El Modelo de Negocio de Correos Chile, establece un sistema de retroalimentación orientado y soportado por sistemas de información transaccionales, sistemas de gestión y diversas plataformas informáticas, las instancias de revisión cuentan con información estructurada a través de la cual se inicia el proceso de retroalimentación organizacional.

A través de talleres de trabajo se realizan revisiones y actualizaciones de los contenidos de las actividades de la planificación de la empresa (Directorio y cuerpo de 
Gerentes), esto les permite identificar aquellos puntos que requieren ser reorientados o trabajados para una mejor consecución de objetivos, metas y resultados.

\section{Tabla 3. Comparación de Elementos en el Sistema de Retroalimentación}

\begin{tabular}{|l|l|l|l|l|l|}
\hline \multicolumn{7}{|c|}{$\begin{array}{c}\text { Modelo } \\
\text { Deming }\end{array}$} & \multicolumn{1}{|c|}{$\begin{array}{c}\text { Modelo } \\
\text { Baldrige }\end{array}$} & $\begin{array}{c}\text { Modelo } \\
\text { EFQM }\end{array}$ & \multicolumn{1}{c|}{$\begin{array}{c}\text { Modelo } \\
\text { Iberoamericano }\end{array}$} & $\begin{array}{c}\text { Modelo } \\
\text { Correos } \\
\text { Chile }\end{array}$ \\
\hline Elanificar & Estrategia & Enfoque & Enfoque & Información \\
\cline { 2 - 6 } & Elementos & Despliegue & Estrategia & Desarrollo & Enfoque \\
\cline { 2 - 5 } & Revisión & Despliegue & $\begin{array}{l}\text { Evaluación y Re- } \\
\text { visión }\end{array}$ & Estrategia \\
\hline Actuar & & $\begin{array}{l}\text { Evaluación y } \\
\text { Revisión }\end{array}$ & & Despliegue \\
\hline & & & & Revisión \\
\hline
\end{tabular}

Fuente: Elaboración propia basada en Nieto C. y Ros McDonnell.

\section{ANÁlisis COMPARATIVO: CRITERIOS}

Es reconocible que todos los modelos de calidad se estructuran en función de la importancia del liderazgo para la consecución de la excelencia final. Los modelos EFQM e Iberoamericano, poseen prácticamente los mismos criterios.

El criterio de personal también es reconocido en todos los modelos, en el Modelo Baldrige como se lo visualiza a través del enfoque en los recursos humanos y en el modelo Deming dentro de los criterios de cooperación interna y aprendizaje, salvo que este modelo no considera el criterio de política y estrategia que si consideran explícitamente en los otros modelos. El Deming solo recoge el criterio de gestión de procesos pero no posee ningún criterio de enfoque al cliente aunque sí se podría decir que dentro del enfoque de liderazgo se tiene en cuenta como fin la satisfacción del cliente.

El modelo Baldrige se centra en los recursos internos y secundariamente en los recursos externos. Hace hincapié en el saber hacer y la gestión del conocimiento con el fin de guiar la mejora y la competitividad organizativa. 
Los resultados en la sociedad no se recogen en el modelo Deming pero sí en el Baldrige, esto resalta el que los modelos EFQM y Baldrige tienen un carácter más ético y por ende un mayor respeto por su entorno social, en relación al carácter más técnico del Deming.

En cuanto al Modelo de Negocio de Correos Chile, se puede decir que tienen claramente definidos ocho criterios que orientan el trabajo de la empresa. Para una mayor ilustración se muestra el siguiente cuadro comparativo.

\section{Tabla 4. Criterios en los que se Basan los Modelos}

\begin{tabular}{|c|c|c|c|c|c|}
\hline $\mathrm{N}^{\mathrm{a}}$ & $\begin{array}{l}\text { Modelo } \\
\text { Deming }\end{array}$ & $\begin{array}{l}\text { Modelo } \\
\text { Malcom } \\
\text { Baldrige }\end{array}$ & $\begin{array}{l}\text { Modelo } \\
\text { EFQM }\end{array}$ & $\begin{array}{c}\text { Modelo } \\
\text { Iberoamericano }\end{array}$ & $\begin{array}{l}\text { Modelo } \\
\text { Correos Chile }\end{array}$ \\
\hline 1 & $\begin{array}{l}\text { Liderazgo } \\
\text { visionario }\end{array}$ & Liderazgo & Liderazgo & $\begin{array}{l}\text { Liderazgo } \\
\text { y Estilo de } \\
\text { Dirección }\end{array}$ & Liderazgo \\
\hline 2 & $\begin{array}{l}\text { Cooperación } \\
\text { interna y } \\
\text { externa }\end{array}$ & $\begin{array}{l}\text { Planificación } \\
\text { Estratégica }\end{array}$ & $\begin{array}{l}\text { Política y } \\
\text { Estrategia }\end{array}$ & $\begin{array}{l}\text { Política y } \\
\text { Estrategia }\end{array}$ & $\begin{array}{l}\text { Planificación } \\
\text { Estratégica }\end{array}$ \\
\hline 3 & Aprendizaje & $\begin{array}{l}\text { Enfoque al } \\
\text { cliente y al } \\
\text { mercado }\end{array}$ & Personas & $\begin{array}{l}\text { Desarrollo de } \\
\text { personas }\end{array}$ & $\begin{array}{l}\text { Enfoque en el } \\
\text { cliente }\end{array}$ \\
\hline 4 & $\begin{array}{l}\text { Gestión de } \\
\text { procesos }\end{array}$ & $\begin{array}{l}\text { Análisis y } \\
\text { dirección del } \\
\text { conocimiento }\end{array}$ & $\begin{array}{l}\text { Alianzas y } \\
\text { recursos }\end{array}$ & $\begin{array}{l}\text { Recursos y } \\
\text { asociados }\end{array}$ & $\begin{array}{l}\text { Enfoque en el } \\
\text { recurso humano }\end{array}$ \\
\hline 5 & $\begin{array}{l}\text { Mejora } \\
\text { continua }\end{array}$ & $\begin{array}{l}\text { Enfoque en } \\
\text { los recursos } \\
\text { humanos }\end{array}$ & Procesos & Clientes & $\begin{array}{l}\text { Gestión de } \\
\text { procesos }\end{array}$ \\
\hline 6 & $\begin{array}{l}\text { Satisfacción } \\
\text { del } \\
\text { empleado }\end{array}$ & $\begin{array}{l}\text { Gestión de } \\
\text { procesos }\end{array}$ & $\begin{array}{l}\text { Resultados en } \\
\text { los clientes }\end{array}$ & $\begin{array}{l}\text { Resultados en } \\
\text { los clientes }\end{array}$ & $\begin{array}{l}\text { Información, } \\
\text { análisis y } \\
\text { dirección del } \\
\text { conocimiento }\end{array}$ \\
\hline 7 & $\begin{array}{l}\text { Satisfacción } \\
\text { del cliente }\end{array}$ & $\begin{array}{l}\text { Resultados } \\
\text { económicos y } \\
\text { empresariales }\end{array}$ & $\begin{array}{l}\text { Resultados en } \\
\text { las personas }\end{array}$ & $\begin{array}{l}\text { Resultados en } \\
\text { las personas }\end{array}$ & $\begin{array}{l}\text { Responsabilidad } \\
\text { social y } \\
\text { empresarial }\end{array}$ \\
\hline 8 & & & $\begin{array}{l}\text { Resultados en } \\
\text { la sociedad }\end{array}$ & $\begin{array}{l}\text { Resultados en la } \\
\text { sociedad }\end{array}$ & $\begin{array}{l}\text { Resultados } \\
\text { económicos y } \\
\text { empresariales }\end{array}$ \\
\hline 9 & & & $\begin{array}{l}\text { Resultados } \\
\text { claves }\end{array}$ & $\begin{array}{l}\text { Resultados } \\
\text { globales }\end{array}$ & \\
\hline
\end{tabular}

Fuente: Elaboración propia basada en Nieto C. y Ros McDonnell. 


\section{AnÁlisis Comparativo: Conceptos o Principios Fundamentales}

A decir de Nieto C. y Ros McDonnell, el Modelo Deming cuenta con diferencias más apreciables en sus principios que los otros modelos de calidad, en concreto seis más que el modelo EFQM y tres más que el modelo Baldrige.

Los catorce puntos establecidos en la filosofía Deming, lo llevan a ser un enfoque mucho más técnico en cuanto a las consideraciones a tener en cuenta a la hora de implantar el modelo.

En el siguiente cuadro se presenta el listado de los principios o conceptos que fundamentan los modelos Deming, Baldrige, EFQM e Iberoamericano así como los que sustentan el del Modelo de Negocio de la empresa de Correos Chile. 


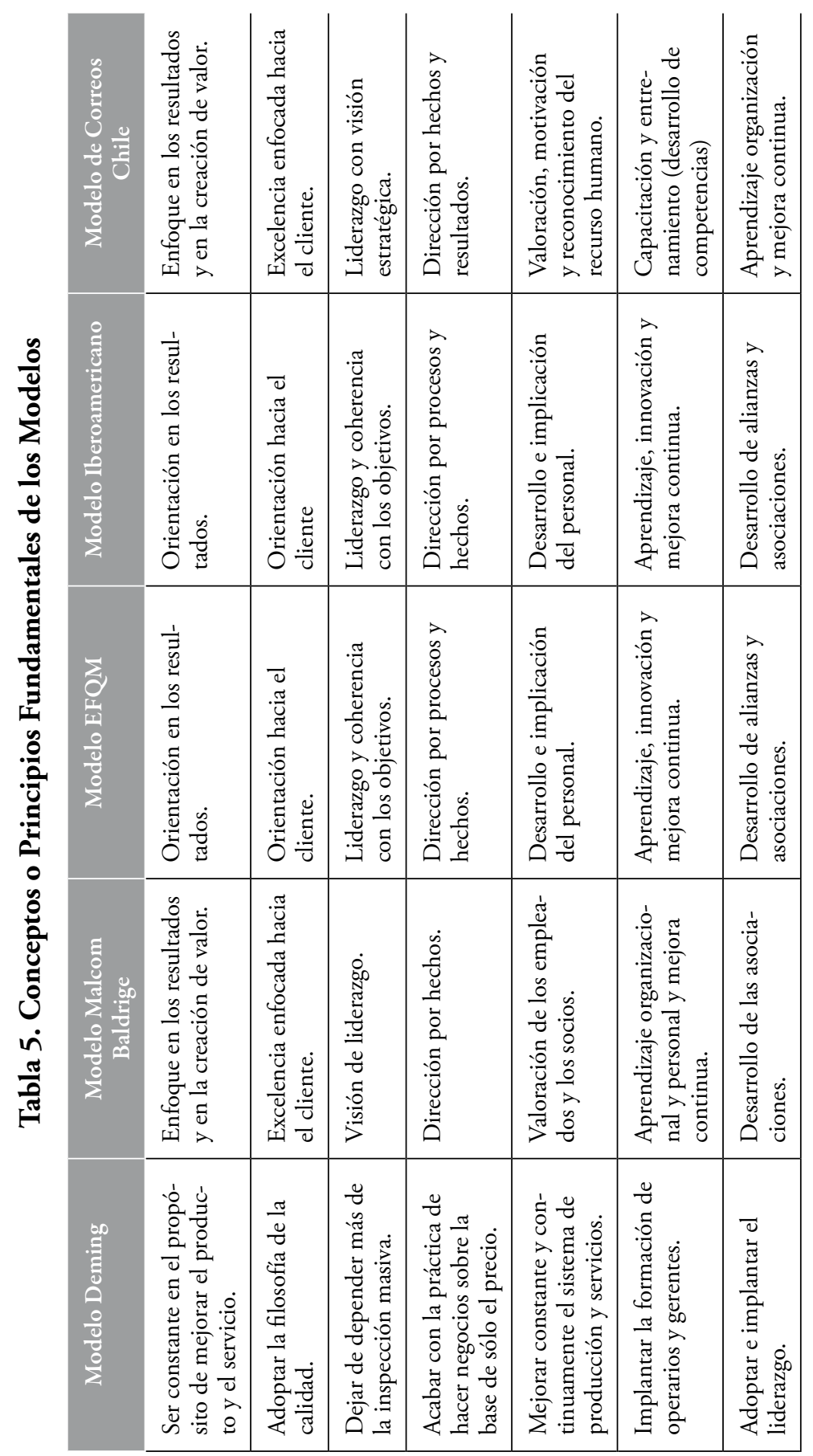




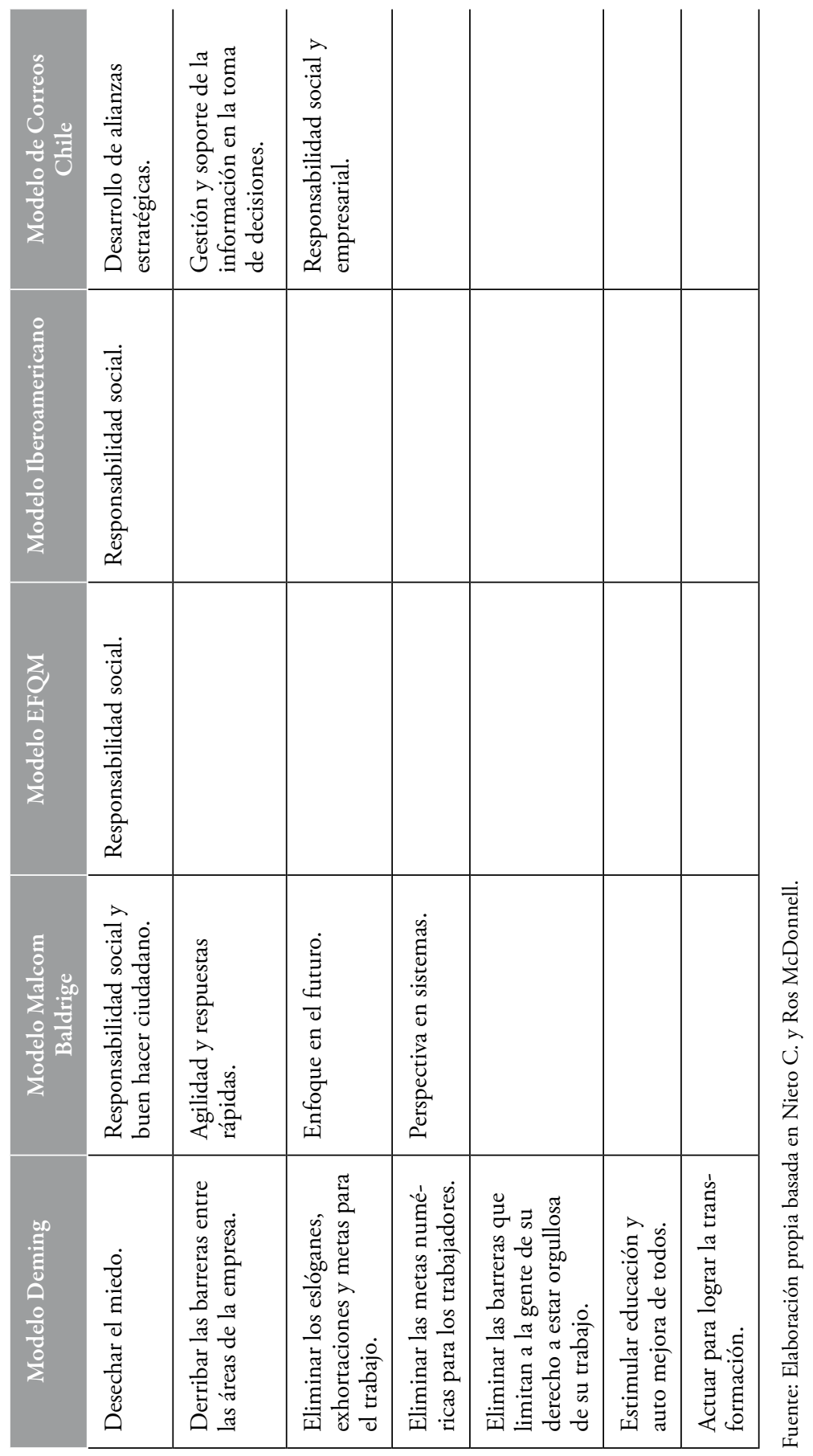




\section{Hallazgos y CONCLUSIONES}

A manera de síntesis se puede decir que los modelos de Gestión de la Calidad expuestos en este apartado, analizados en sus diferentes variables de misión, enfoque, sistema de retroalimentación, criterios y principios fundamentales, representan una filosofía de trabajo empresarial que se proyecta tanto al interior como exterior de la organización, con la finalidad de alcanzar una posición competitiva en términos de satisfacción del cliente, desarrollo de competencias del recurso humano, costos, rentabilidad, calidad del producto o servicio, posicionamiento en el mercado, resultados, buen hacer social, etc. Asumir principios de calidad en la gestión implica una revolución cultural a todo nivel, un cambio de actitud de todo el personal y en todos los niveles de la empresa.

Todos los modelos antes analizados presentan similitudes respecto a los criterios y elementos bajo los cuales sustentan su filosofía o concepto de gestión de calidad, sin embargo cada uno tiene su propio enfoque de sistema del proceso a seguir para hacer tangibles los principios en resultados organizacionales y empresariales, con políticas y estrategias proactivas que permitan anticiparse a las futuras necesidades de mercado y permanecer en este.

Las prioridades de gestión de la Empresa Pública de Correos Chile han cambiado drásticamente durante los últimos años. Antes del 2002 la gestión se basaba fundamentalmente en función de ampliar solamente la cobertura en la distribución postal, producto de las grandes transformaciones y cambios, y la necedad imperiosa de revertir los resultados negativos de la empresa; a partir de este año los representantes del directorio decidieron adoptar una nueva filosofía de trabajo y gestión centrada en mejorar la calidad del servicio, generar productos y servicios con valor agregado para el cliente, contar con recurso humano capacitado y calificado, tomar decisiones sustentados en sistemas de información adecuados y a través de esto lograr un mayor posicionamiento en el mercado y mejores resultados económicos.

Analizando las diversas variables que inciden en el Modelo de Negocio de la empresa Correos Chile, se puede decir que su modelo ha sido guiado por la estructura, filosofía y principios del modelo americano Malcom Baldrige, por orientar su gestión con un enfoque a la creación de valor, satisfacción al cliente, desarrollo del 
recurso humano y dirección de resultados económicos y empresariales. Asimismo se puede observar que el modelo de negocio de la empresa se encuentra sustentado en sólidas políticas de liderazgo, lo que permite tener una visión estratégica de futuro, con estrategias de análisis y gestión de información en la toma de decisiones y dirección del conocimiento.

Los resultados de este estudio indican que la adopción de principios de gestión de calidad de los diversos modelos de excelencia, son una buena decisión económica y responde a los parámetros de fortalecimiento de la Gestión requeridos para el desarrollo de la productividad y la competitividad de Correos Chile.

Correos Chile como empresa del Estado tiene muchas restricciones que juegan en contra al momento de competir en el mercado con empresas privadas de servicio postal que tienen participación en el mercado. Pese a ser una empresa autónoma del Estado la toma de decisiones está sujeta al cumplimiento del Decreto de Fuerza de Ley No 10 (DFL promulgado y publicado el 30 de enero de 1982), Ley Postal en la que una serie de disposiciones limitan la competitividad de la empresa tales como:

- Tarifas máximas para el servicio postal.

- Pesos máximos para la aceptación de piezas postales.

- Régimen impositivo extraordinario (17\% más 40\% sobre utilidades).

- Todas las utilidades van al fisco.

- Rigidez en las políticas de inversión.

La aprobación de la Nueva Ley Postal permitiría que Correos Chile compitiese en igualdad de condiciones y de manera más justa con la competencia. A partir del 2002, Correos Chile como empresa autónoma del Estado ha sabido fijar y plantear sus prioridades de trabajo y de gestión; en este contexto los cambios de gobierno han influido en menor grado pero no han cambiado el rumbo de la orientación estratégica en la gestión como empresa. La continuidad en la línea política que se ha fijado como desafío el tener empresas e instituciones modernas, ha orientado e impulsado a que Correos Chile se inserte bajo esta perspectiva orientada hacia la búsqueda de la competitividad; esto ha permitido que los proyectos planteados por la empresa tengan continuidad en los distintos mandos superiores, lográndose mantener las políticas de mejoramiento continuo en bús- 
queda de la excelencia para convertirse en una empresa que ofrezca calidad y que dé soluciones tanto a sus clientes como a la ciudadanía en general.

La relación con los sindicatos ha evolucionado y mejorado durante los últimos años, los trabajadores y representantes vienen entendiendo y aceptando que las diversas medidas adoptadas por la empresa son por el bien de los trabajadores, por la permanencia de la empresa en el mercado y de ellos. Pese a que algunas medidas tomadas por la empresa son vistas un tanto como no adecuadas para el sindicato (establecimiento permanente de metas, evaluación del personal, etc.), deben ser vistas como una necesidad y no un capricho para poder seguir competiendo con el mercado externo.

Un tema resaltante es que la empresa viene impulsando la carrera funcionaria: existe un importante porcentaje de mandos medios y superiores que se han formado dentro de la empresa, dándose tiraje a lo que es el ascenso del personal al interior de la organización. Esto motiva el desempeño de los trabajadores en sus diferentes líneas de mando.

El método utilizado en la investigación ha permitido realizar el registro y conocimiento del contexto estructural y situacional del proceso de adopción del modelo de negocio en la Empresa Pública Correos Chile, permitiéndonos un entendimiento a profundidad sobre la naturaleza y evolución de las diferentes situaciones y realidades implementadas por la organización al asumir principios de gestión de calidad y cambios adoptados en sus diferentes sistemas y su estructura.

Las limitaciones del estudio han estado básicamente referidas a la ubicación geográfica de los informantes claves. Correos Chile por su naturaleza de empresa pública tiene oficinas o agencias de atención distribuidas en todas las regiones del país, descentralizando la gestión en cuatro zonas: Gerencia Zonal Norte comprende las regiones I, II, III y IV, tiene como sede la ciudad de Antofagasta; Gerencia Zonal Centro comprende las regiones V y VI, tiene como sede la ciudad de Viña del Mar; Gerencia Zonal Sur comprende las regiones VII, VIII y IX, tiene como sede la ciudad de Concepción; Gerencia Zonal Austral comprende las regiones X, XI y XII, tiene como sede la ciudad de Puerto Montt; y la Región Metropolitana. 
Otra limitante encontrada fue la disponibilidad de tiempo de los informantes clave ya que durante el proceso de levantamiento de información y entrevistas algunos de ellos se encontraban en capacitación de la empresa.

A través de este estudio si ciertamente no se tiene como finalidad hacer una generalización de los resultados para hacer inferencia a una población, sí se plantea la utilización de esta investigación para dar orientaciones, pistas y pautas de actuación a empresas enmarcadas en contextos similares que tratan de implementar de manera exitosa estas prácticas en sus sistemas de gestión, así como utilizar este trabajo como aporte al conocimiento de futuras investigaciones en este campo.

\section{REFERENCIAS Bibliográficas}

Albacete, C. A. (2004). Calidad de Servicio en Alojamientos Rurales. España: Editorial Universidad de Granada.

Albacete, C. A.; Fuentes, M. M. y Lloréns, F. J. (2007). “Service Quality Measurement in Rural Commodation”. Annals of Tourism Research. Vol. 34, No 1, pp. 45-35.

Albretch, K. (1994). Todo el Poder al Cliente: el nuevo imperativo de la calidad del servicio. Barcelona: Paidós.

Barzelay, Michael (1992). Breaking Throught Bureacracy, A New Vision for Managing in Government. Los Angeles: University of California Press.

Beltrami M. (1992). "Qualità e pubblica amministrazione”. Economia e diritto del terziario No 3, pp. 669-781.

Castelló Taliani E, y Lizcano Álvarez, J. (1998). La mejora de las entidades públicas en la Europa del Euro. Papel del sistema ABC/ABM. Espańa: AECA.

Correos Chile (2001 - 2003; 2004, 2005, 2006, 2007). Informes de Gestión. Santiago.

Sistema de Empresas Públicas (2008). Memoria Anual, Correos Chile. Santiago: SEP.

CLAD (1999). "Una Nueva Gestión Pública para América Latina”. Revista del CLAD, Reforma y Democracia, № 13.

Crosby, P. (1979). Quality is free. New York: Editorial McGraw-Hill Book Co.

Crozier, Michel (1997). "La transición del paradigma burocrático a una cultura de gestión pública”. Revista del CLAD, Reforma y Democracia No 7, pp. 9-18. 
Deming, E. (1989). Quality, Productivity and Competitive Position. Center for Advanced Engineering Study. Cambridge, Mass: Massachusetts Institute of Technology.

Drucker, Peter (1985). La Innovación y el empresario innovador. México: Ed. Edhasa.

EFQM (1988). Fundación Europea para la Gestión de la Calidad, http://www.efqm.com

Fundación Iberoamericana (1999). Fundación Iberoamericana para la Gestión de la Calidad, en: http://www.fundibeq.com

Galindo, C. (1998). Técnicas de investigación en sociedad cultura y comunicación. México: Addisión Wesley-Parson.

Garde, J. (2005). "Nueva gerencia pública: evaluación, calidad e innovación”. Ponencia X Congreso Internacional del CLAD sobre la Reforma del Estado y de la Administración Pública, Chile.

Guerrero, Omar (2004). "El mito del nuevo "Management" público". Revista Venezolana de Gerencia, Año 9, No 25, pp. 9-52.

Gurú, C. (2003). “Tailoring e-service quality through CRM. Managing Service Quality”. Vol. 13, No 6, pp. 520 - 531.

Kvale, S. (1996). Interviews: An Introduction to Qualitave Research Interviewing. Thousand Oaks, CA: Sage.

López, Andrea (2002). "La Nueva Gestión Pública: Algunas Precisiones para su Abordaje Conceptual”. Serie I, Desarrollo Institucional y Reforma del Estado Doc. No 68.

Malcolm Baldrige (1987). The Baldrige Model, disponible en: http://www.baldrige.com (accesado en octubre 2009).

Metcalfe, L. (1999). "La gestión Pública: de la imitación y la innovación”, en Losada i Madorrán (editor), ¿De Burócratas a Gerentes? Washington D. C.: Banco Interamericano de Desarrollo.

Nieto N. y Ros McDonnell L. (2006). “Comparación entre los modelos de gestión de Calidad Total: EFQM, Gerencial de Deming, Iberoamericano para la Excelencia y Malcom Baldrige. Siruacion frente a la ISO 9000". Ponencia X Congreso de Ingeniería de Organización, Valencia.

OCDE (2005). Reporte Anual, disponible en www.ocde.org

Oria, Elizabeth (2008). “Cambios en la Gestión de Empresas Públicas Uruguayas en la Década de 1990”. Estado, Gobierno y Gestión Pública, Revista Chile de Administración Pública No 11, pp. 89-115. 
Pollit, Cristopher y Bouckaert, Geert (2004). Public Management Reform: A Comparative Analysis. Oxford: Oxford University Press.

Rialp, A. (1998): "El método del caso como técnica de investigación y su aplicación al estudio de la función directiva”. Ponencia IV Taller de Metodología ACEDE, La Rioja.

Savoie, Donald (1995). "What is wrong with the New Public Management". Canadian Public Administration Review, Vol. 38, No 1, pp. 112-121.

Yin, Robert (1994). Case study research. Design and methods. Beverly Hills, CA: Sage Publications. 